\title{
Heart rate and nictitating membrane classical discrimination conditioning in rabbits under delay versus trace procedures'
}

\author{
A. L. MEREDITH AND N. SCHNEIDERMAN \\ UNIVERSITY OF MIAMI
}

Each of 32 Ss received 1 adaptation, 7 acquisition, and 2 extinction sessions in a 2 by 2 factorial design with delay and .25 sec trace procedures as one dimension and interstimulus intervals of $.35 \mathrm{sec}$ and $1.00 \mathrm{sec}$ as the other. The chief findings were that: (a) heart rate (HR) responses conditioned more rapidly than nicitating membrane (NM) responses; ( $b$ ) HR performance was directly related and NM performance inversely related to interstimulus interval; and (c) the trace procedure debilitated NM performance at the $1.00 \mathrm{sec}$ interval.

The purpose of the present experiment, which jointly and simultaneously conditioned nictitating membrane (NM) and heart rate (HR) responses in rabbits, was to study the differences in performance among response systems as a function of varying interstimulus intervals (ISI) and delay $\nabla 8$ trace conditioning procedures.

Previous classical conditioning experiments comparing the effects of delay vs trace conditioning at different ISIs have reported contradictory findings. Church \& Black (1958) examined a HR response in dogs at ISI up to 20 sec and found no essential differences between procedures. In contrast, Ellison (1964), investigating salivary discrimination conditioning in dogs, observed that the trace procecure was inferior to the delay at a $16 \mathrm{sec}$ IS, although there were no differences between procedures at an ISI of $8 \mathrm{sec}$. Finally, Schneiderman (1966) assessed NM conditioning in rabbits at ISI of .25 to $2 \mathrm{sec}$, and reported that the trace procecture resulted in poorer conditioning than the delay at these intervals.

Nethod

One dimension of a 2 by 2 factorial design, numbering eight rabbits per cell, consisted of .35-sec or 1.00sec ISI; the other dimension consisted of a .25-sec trace procedure, or a delay procedure in which the CS and US offset simultaneously. All Ss received one day of adaptation to 550 and $3400 \mathrm{cps}$ tones at $78 \mathrm{~dB}$ SPL followed by seven days of classical discrimination conditioning and two days of experimental extinction to both tones. In each acquisition session the $S$ received 80 trials at an intertrial interval of $85 \mathrm{sec}$. Thirtyfive presentations of one frequency tone were followed by a $3 \mathrm{~mA}, .3 \mathrm{sec}$ duration ac shock delivered to the Ss' right eyelids through two hooks which held the lids open; five presentations of the same frequency tone presented alone served as test trials for the reinforced tone. Of the 40 presentations of the never reinforced tones, five presentations served as test trials.
The conditioning apparatus, methods of NM and HR recording, and manner in which each $S$ was restrained within a Plexiglas box have previously been described (Schneiderman \& Gormezano, 1964; Schneiderman, Smith, Smith, \& Gormezano, 1966). Measures of HR were obtained on test trials by dividing the duration of the 25 successive heart beats occurring after tone onset by the duration of the 25 heart beats preceding tone onset; NM responses also were recorded on test trials. Results

Figure 1 indicates that only the 1.00 sec trace group failed to reveal a high level of NM conditioning. A two-way analysis of variance conducted upon the cumulated NM responses in acquisition indicated a significant source of variation $(p<.01)$ between the .35 and 1.00-sec ISI groups, but the differences between the delay and trace procedures were not reliable. However, a significant interaction ( $p<.01$ ) between dimensions reflected the fact that the trace procedure revealed both the highest and the lowest levels of conditioning. A similar two-way analysis of variance conducted upon the cumulated NM responses in extinction reverled significant differences $(p<.01)$ in the trace vs delay and ISI dimensions as well as in the interaction between dimensions. Analyses of variance conducted separately upon cumulated discrimination scores in acquisition and extinction falled to reveal any differences in discrimination among groups.

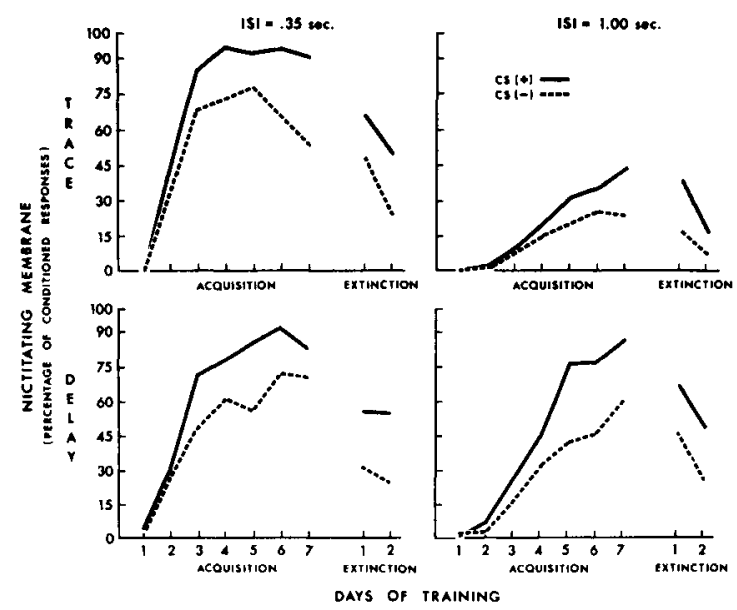

Fig. 1. Percentages of nictitating membrane responses during test trials for each ISI group under delay and trace procedures. 


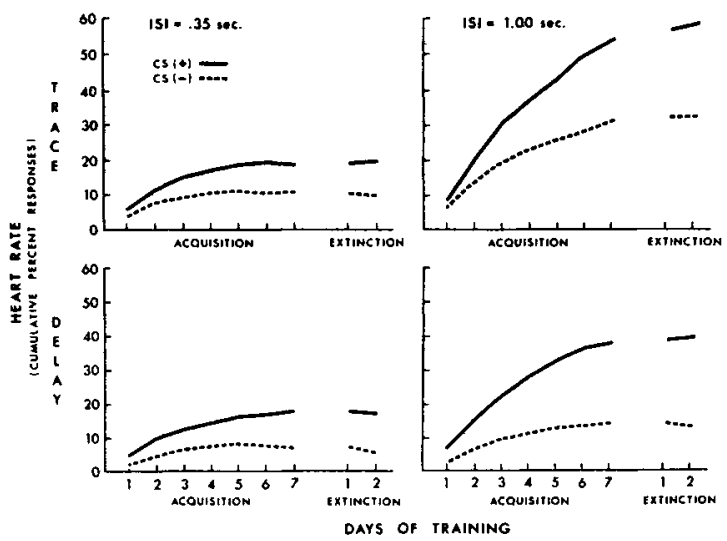

Fig. 2. Cumulative mean daily test trial percentages of heart rate deceleration from baseline for each ISI group under delay and trace procedures.

Two-way analyses of variance conducted upon the cumulated percentage changes of HR decreases from baseline in acquisition and extinction (shown in Fig. 2) confirmed that the $1.00-s e c$ IST groups revealed greater amounts of differential conditioning and higher levels of $\mathrm{HR}$ responding to the CS+ than the .35-sec groups ( $p<.01)$, whereas the differences in $H R$ responding between the delay and trace procedures were not reliable.

A one-way analysis of variance conducted upon the cumulated HR responses in the first acquisition session indicated a reliable $(p<.01)$ difference between responding to the CSt and the CS-. In contrast, there were almost no NM responses recorded during the first session in any group, and consequently no reliable differences in responses to the reinforced and nonreinforced stimuli.

\section{Discussion}

The differences observed between response systems in rate of CR acquisition, shape of the IST function, and performance under the trace procedure in the present experiment are compatible with Ebel \& Prokasy's (1963) time discrimination hypothesis. According to this formulation, strength of conditioning is related to the extent to which a particular ISI favors $\mathrm{S}$ making a time discrimination which permits kinesthetic feedback from a CR to occur in proximity with a US.

Since duration of a CR would thus appear to be directly related to the probability of overlapoccurring,
Schnelderman (1966) predicted that long CR duration response systems such as HR should reveal more rapid conditioning across a greater range of ISIs than a short $\mathrm{CR}$ duration response system such as NM. Verification of these predictions was provided by VanDercar \& Schneiderman (1967) who found that: (a) HR responses conditioned more rapidly than NM responses; and (b) HR, but not NM responses, conditioned reliably at a $6.75 \mathrm{sec}$ ISI. In addition, it was observed in both the VanDercar \& Schneiderman and the present study that HR but not NM conditioning was debilitated at ISI under one sec. Presumably this occurred because the US truncated the long duration HR response at short intervals thus interfering with S's learning to time his responses.

The present experiment confirmed Schneiderman's (1966) observation that a trace procedure debilitated NM conditioning, and Church \& Black's (1958) finding that the trace procedure did not debilitate HR conditioning. In terms of the time discrimination hypothesis it would appear that the introduction of a $.75 \mathrm{sec}$ temporal gap between CS offset and US onset in the trace procecure increased the difficulty of $S^{\prime} s$ appropriately placing the short duration NM responses in the CS-US interval. In contrast, in the $H R$ response system the $\mathrm{S}$ had only to respond as quickly as possible after CS onset in order for his response to overlap the US.

\section{References}

CHURCH, R. N., \& BLACK, A. H. Conditioned heart rate and CS-US interval. J. comp. physiol. Psychol, 1958, 51, 478-482.

EBEL, H. C., \& PROKASY, W. F. Classical eyelid conditioning as a function of sustained and shifted interstimulus intervals. J. exp. Psychol, $1963,65,52-58$.

ELLISON, G. D. Differential salivary conditioning to traces. J. comp. physiol Psychol, 1964, 57, 373-420.

SCHNEIDERMAN, N. Interstimulus interval function of the nictitating membrane response of the rabbit under delay versus trace conditioning. J. comp. physiol Psychol, 1966, 397-402.

SCHNEIDERMAN, N., \& GORMEZANO, I. Conditioning of the nictitating membrane of the rabbit as a function of CS-US interval.J. comp. physiol Psychol, 1964, 57, 188-195.

SCHNEIDERMAN, N., SMITH, M. C., SMITH, A. C., \& GORMEZANO, I. Heart rate classical conditioning in rabbits. Psychon. Sci, 1966, 6, 241-242.

VanDERCAR, D. H., \& SCHNEIDERMAN, N. Interstimulus interval functions in different response systems during classical discrimination conditioning of rabbits. Psychon. Sci, 1967, 9, 9-10.

Note

1. This research was supported by NSF Grant GB-5307. 